\title{
Recurrent balloon rupture during Transcatheter Aortic Valve Replacement (TAVR) - a challenge to the Hybrid team
}

\author{
Imthiaz Manoly*, Andrew Brazier, Ahmed Mustafa, Vasim Farooq, Ragheb IR Hasan, Douglas G Fraser \\ From World Society of Cardiothoracic Surgeons 25th Anniversary Congress, Edinburgh \\ Edinburgh, UK. 19-22 September 2015
}

\section{Background/Introduction}

Transcatheter aortic valve implantation is at present reserved for patients with severe aortic valve stenosis who are high risk for conventional surgery or surgical access is difficult due to heavily calcified aorta (porcelain aorta). However the calcium spikes at the root could pose a problem during balloon valvuloplasty resulting in rupture of the balloon.

\section{Aims/Objectives}

Our objective was to assess patients who have heavy calcification of the aortic valve and aortic root and recommend measures to overcome the difficulty in the deployment of transcatheter valve.

\section{Method}

After careful assessment including computed tomography of the arterial tree, it was decided that the best approach was trans-aortic as the heavy calcification would not allow the trans-femoral or transapical approach feasible. Despite this, there were ruptures of the balloon twice during valvuloplasty which were retrieved at the ascending aorta.

\section{Results}

We were successful in deploying a $26 \mathrm{~mm}$ Edwards Sapien XT through trans-aortic approach after two failed attempt due to rupture of the balloon during valvuloplasty. The patient also had an off pump coronary artery bypass grafting to the right coronary artery. The patient was discharged home after a week.

\section{Discussion/Conclusion}

TAVR balloon rupture is an uncommon complication; it could result when the balloon expands against a sharp spike of calcium as it was in this case. The issue of retrieving the balloon could be complicated in such scenarios particularly if the access was through a transfemoral route. In such a situation the retrieval of the balloon and sheath system would require pulling back the delivery system into the descending aorta and performing a potentially complicated unplanned ilio-femoral vascular exploration. On the other hand, the appearances of sharp spike-like calcifications would favour the trans-aortic approach where retrieval of the system if the balloon ruptures is more straightforward. Pre-operative Computed tomography is very essential in selecting the best access for trans catheter valve.

Published: 16 December 2015

doi:10.1186/1749-8090-10-S1-A316

Cite this article as: Manoly et al: Recurrent balloon rupture during Transcatheter Aortic Valve Replacement (TAVR) - a challenge to the Hybrid team. Journal of Cardiothoracic Surgery 2015 10(Suppl 1):A316. 